\title{
Referencing technique for phase detection in eddy current evaluation
}

\author{
N.A. Nadzri', M.M Saari' ${ }^{1}$ M.A.H.P Zaini1 and Z.A. Aziz' ${ }^{1}$ \\ ${ }^{1}$ Faculty of Electrical \& Electronics Engineering Technology, Universiti Malaysia Pahang, 26600 Pekan, Pahang, Malaysia
}

\begin{abstract}
This paper presents an eddy current testing (ECT) method with a different type of referencing technique for crack detection in carbon steel plate by using two Anisotropic Magnet Resistance (AMR) sensors to detect and evaluate the artificial cracks and to study the output signal's characteristics of each technique. The experiment setup of the magnetic scanning device and the measurement mode is included. Based on these three techniques that have been used, it shown that differential technique with the condition of the required reference signal must be dynamic from one point to one point was the best way to implement in the ECT method. The line scanning results indicate that performing these three techniques can be used to estimate the position of the slits, however, for 2-D mapping, it is shown that the differential technique is a preferable technique where it can remove the noise response, and at the same time, it generates a clear image of the crack. It is predicted that the developed ECT probe using a differential technique can be used as a technique to estimate the characteristic of defects in a metal plate.
\end{abstract}

ARTICLE HISTORY

Received: 30th Mar. 2021

Revised: $05^{\text {th }}$ Oct. 2021

Accepted: $21^{\text {st }}$ Oct. 2021

\section{KEYWORDS}

Eddy current testing;

anisotropic magnet;

resistance;

crack;

steel.

\section{INTRODUCTION}

Non-destructive tests (NDT) are commonly used to detect structural defects, surface changes, the development of defects or other defect abnormalities, to evaluate the thickness of materials and coatings, and to determine other industrial product characteristics [1]. All the materials and structures can be inspected without being harmed by applying NDT since the procedure is contactless with the component [2]. Frequently, NDT is applied in industries to prevent failure and accidents by making sure that all the structure is in good condition to work on. Furthermore, while the product undergoes the processing technique of NDT, without affecting its performance, NDT can increase the degree of reliability of the product. In industries, nondestructive testing (NDT) is commonly used [3].

Eddy current testing (ECT) [4] is one of the relevant electromagnetic NDT techniques technology to improve the quality of the product especially for metal structure [5]. Eddy current is created from the process of electromagnetic induction. For ECT method, the alternating current flows in the excitation coil generate the magnetic field. [6]. If there is any conductive material brought closer to the coil, the eddy current will be induced in the sample and the flaws on the sample will make the changes of amplitude and distribution of eddy currents [7]. Then the disruption caused by the irregular of the material is measured by the induction coils [8] or magnetic sensors $[9,10]$. Plenty of ECT probes were developed with various types, designs, and sizes so that they fit and are easy to use in the desired applications. To make sure that the probe will fit with the testing area and purpose, the types of the probe operation needs to be studied first. The mode of operation divided into three which is absolute, differential [11] and reflection [12].

In this study, the new differential eddy current testing sensor is suggested which consists of two magnetic sensors with the excitation coil. In the beginning, this research started with an AMR sensor [13,14] which means it only contained a single absolute probe. This sensor module was used in the first attempt of the study to integrate the magnetic field sensor into the ECT probe but when the scanning process was conducted, it is found that the measured signal contained metal and background signals which could be assumed as noises. By using one sensor, it was observed that the background signals interfered during the scanning process. For this reason, to make sure the background signal fades away, a new method is proposed by using two magnetic sensors [15]. This method is called as differential analysis using dual-channel sensor probe [16]. The output signal is analyzed using a component of magnetic vector, $\vec{B}$ which is Bz through the lockin detection. In the lock-in detection, a reference signal for the phase sensitive detection is required.

In a conductive material, if the magnetic and the physical properties are in the same conditions, then the reference signal will remain the same at all locations of the material. Unfortunately, if there is a distribution in the magnetic properties in the material, then the reference signal will be different in each location since the eddy current is related to conductivity, $\sigma$ and permeability, $\mu$ of the material. The reference signal is used to measure the phase delay of the eddy currents. Due to this, it can cause false detection even there is no crack in the material if the phase signal is incorrect.

To improve this kind of obstacle, instead of using location as the reference technique, sensor 1 is proposed as the reference signal which means that the reference signal will change according to location of sensor 1. By this technique, the dependency of distribution of the magnetic properties can be reduced. However, the disadvantage of using this method is the data of the absolute reading and the local data of the properties of magnetic and physical properties of the

sample are lost because of the differential technique. But in this study, this will not be an issue because the interest of this study is to identify the change of eddy currents due to crack or defects in materials. 
During line scanning of the cracks, the probe runs in the direction of the $x$-axis. This probe works as detection of eddy current when the eddy current stumbles upon something else. The detected signal will change by following the flow of the current. For an explanation, a current that is in $y$-direction will produce two magnetic fields in $x$ - and $z$-directions, $B x$ and $B z$, respectively. If the crack in the material is in $y$-direction, this will block and redirect the current to the $y$-direction. According to Maxwell equation, the $y$-directed current can be determined by measuring the magnetic fields that are perpendicular to current.

$$
\nabla \times \frac{\vec{J}}{\sigma}=\frac{d}{d t} \vec{B}_{a p p l y}
$$

In this study, an ECT probe with two AMR sensors is used to detect the presence of defects. The objective of this study is to make a comparison between three different reference technique. For detection, a $2 \mathrm{~mm}$ thick carbon steel plate is used to analyse and evaluate the change of eddy currents. This paper discusses details of the results of the investigation towards the different reference technique of magnetic field distribution.

\section{EXPERIMENTAL SETUP}

\section{Signal Reference}

During the process of scanning, a magnetic response from sensor $1\left(\mathrm{~S}_{1}\right)$ will produced when the Ac magnetic field applied to the conductive samples. The signal produce from $S_{1}$ contains an enormous amount of magnetization signal and small eddy current induced signal. Two separate these two components, a lock in amplifier is used where the real part was set for magnetization signal and the imaginary part is for eddy current signal. Therefore, by using signal referencing where $S_{1}$ as the reference signal, the intensity of the differential vector $S_{1}-S_{2}$ is figured. The vector for sensor $1, S_{1}$, and sensor 2, $S_{2}$, can be illustrated as in Figure 1. Since the large magnetization signal is abated by the means of difference between the sensors, the phase delay of the small eddy current can be identified.

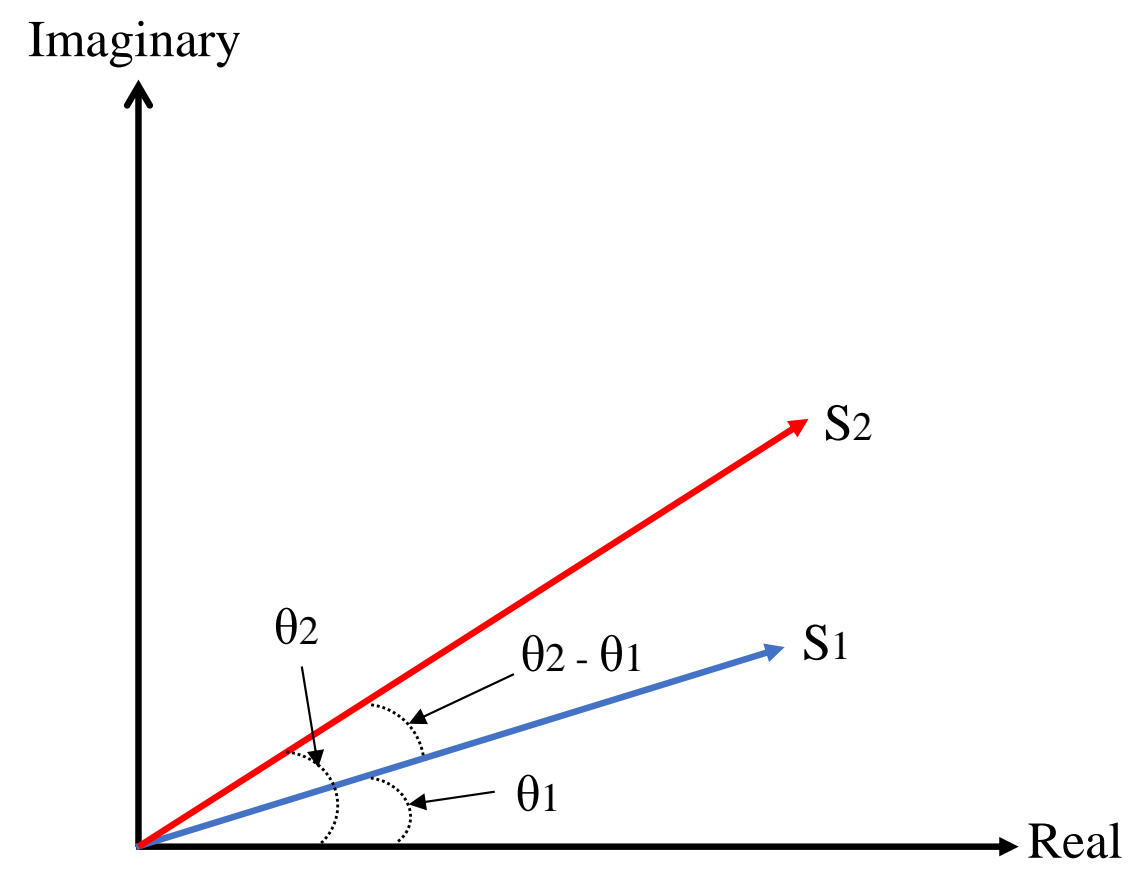

Figure 1. Differential vector S1 and S2 of the measured magnetic signals

During experimental setup, the $X$ and $Y$ is defined by referencing the resultant, $R$ with the output signal from sensor $1, S_{1}$. Vector corrections need to be done to ensure the $X$ and $Y$ to be referenced with $S_{I}$. The mathematical expressions of both $S_{1}$ and $S_{2}$ are as follows,

$$
\begin{aligned}
& S_{1}=X_{1}+Y_{1} \\
& S_{2}=X_{2}+Y_{2}
\end{aligned}
$$

Next, the expressions can be further elaborated as follows, 


$$
\begin{aligned}
& S_{1}=\sqrt{X_{1}^{2}+Y_{1}^{2}} \cos \theta_{1}+j \sqrt{X_{1}^{2}+Y_{1}^{2}} \sin \theta_{1} \\
& S_{2}=\sqrt{X_{2}^{2}+Y_{2}^{2}} \cos \theta_{2}+j \sqrt{X_{2}^{2}+Y_{2}^{2}} \sin \theta_{2}
\end{aligned}
$$

The mathematical expressions of both $X$ and $Y$ with referenced to $S_{l}$ can be shown from the equation below:

$$
\begin{aligned}
& \operatorname{Xref}_{1}=\sqrt{X_{2}^{2}+Y_{2}^{2}} \cos \left(\theta_{2}-\theta_{1}\right)-\sqrt{X_{1}^{2}+Y_{1}^{2}} \sin \left(\theta_{1}-\theta_{1}\right) \\
& \operatorname{Yref}_{1}=\sqrt{X_{2}^{2}+Y_{2}^{2}} \sin \left(\theta_{2}-\theta_{1}\right)-\sqrt{X_{1}^{2}+Y_{1}^{2}} \sin \left(\theta_{1}-\theta_{1}\right)
\end{aligned}
$$

To make sure that both sensors are to be referenced to the $S_{l}$, the phase of both sensors should be subtracted with the phase of $S_{l}$, which is $\theta_{l}$.

\section{Sample Preparation}

Artificial slits are laser-engraved on a 2-mm galvanized carbon steel plate to test the efficacy of the ECT probe. Galvanized carbon steel plate has been widely used in industries due to its excellent rust resistance. The galvanized steel plate, on the other hand, is a ferromagnetic material, and a recent paper stated that ECT was impossible to handle on ferromagnetic materials since magnetic field responses involve both eddy current and strong magnetization signals [17]. The test samples are 2-mm thick with different slit-like artificial cracks. Few different depth are prepared to analyze the crack detection efficiency. A fibre laser marking machine is used to create the artificial slits.

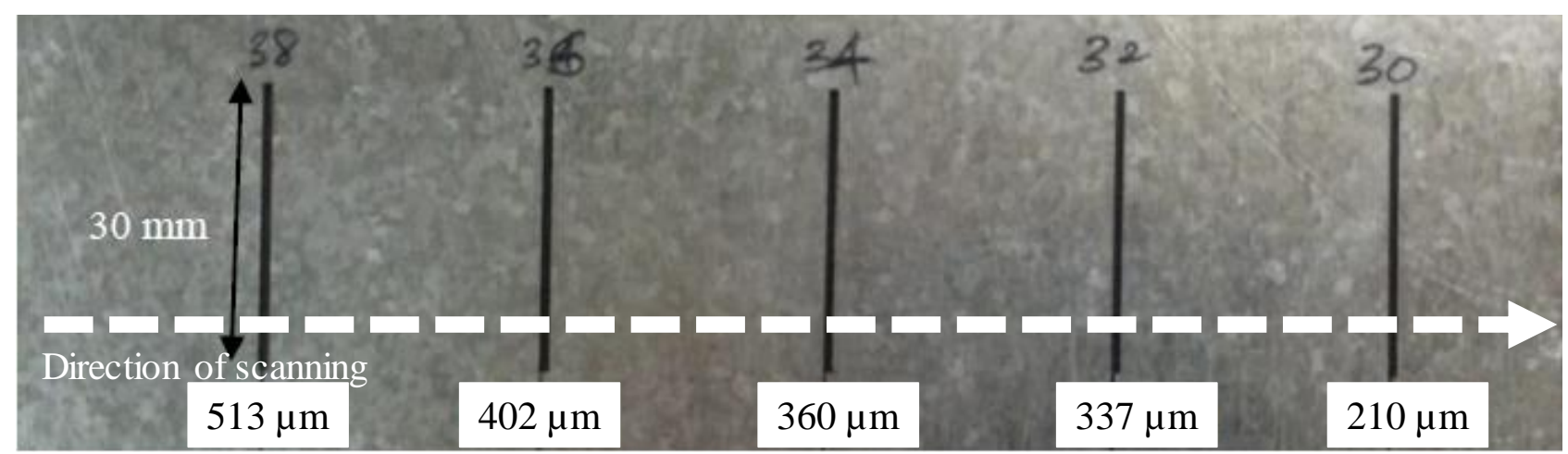

Figure 2. Differential vector S1 and S2 of the measured magnetic signals. Artificial slits (defects) of depth from 210 $\mu \mathrm{m}$ to $513 \mu \mathrm{m}$ on a galvanized carbon steel plate

\section{ECT Probe}

Probes of eddy currents come in many shapes and sizes. In fact, the probes for ECT technique can be customized according to the application which is this factor is one of the major advantages in ECT technique. To choose the best configuration of the probe, one must study the way of the coil package to best fit with the testing area. There are few types of operation of the probe in ECT. For a selection of operation mode, it must refer to the way of the coils are wired and interface with the test equipment. The ECT probe's main purpose in this experiment is to detect a surface defect. Therefore, a compact ECT probe is beneficial because it can be used to evaluate cracks with a tiny or complex shape. Since the AMR sensor is small, it can be used to improve the ECT probe while also solving the magnetic noise problem in conductive materials.

In this experiment, to form a differential technique, two anisotropic magnetoresistance (AMR) (Honeywell HMC1001) sensors are used with each in absolute mode. Since this research aims to detect sub-millimetre crack surface defects, the usage of small sensor is needed to improve the localization performance of the crack detection. Usually, most of low field magnetic sensors will be influenced by the large magnetic disturbing fields. A Set/ Reset strap in the sensor is used in order to restore the sensitivity of the AMR elements. Because of that, a dual-channel sensor probe is developed. To detect the differential signal, the two AMR sensors are connected and separated by a 4-mm baseline. To generate a magnetic field, an excitation coils is used with dimensions of $11.5 \mathrm{~mm} \times 11.5 \mathrm{~mm}$. Using the prepared metal plate, the ECT probe is placed $1 \mathrm{~mm}$ above the plate during the scanning process. Figure 3 shows the arrangement of the two sensors where it plays an important role during the scanning process. This arrangement can detect the difference of magnetic fields between two locations to achieve the differential technique. 
(a)

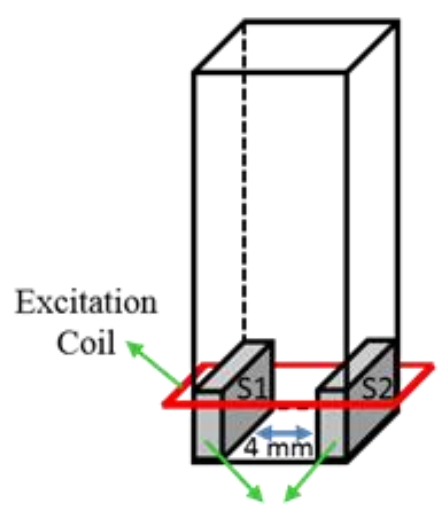

AMR Sensors (b)

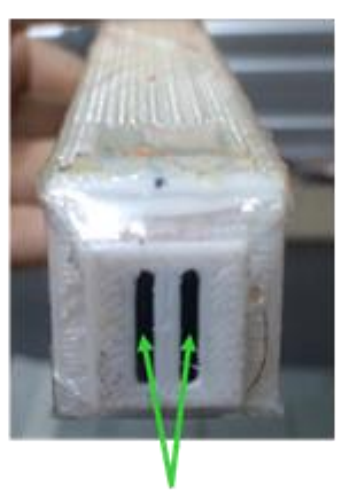

AMR sensors (c)

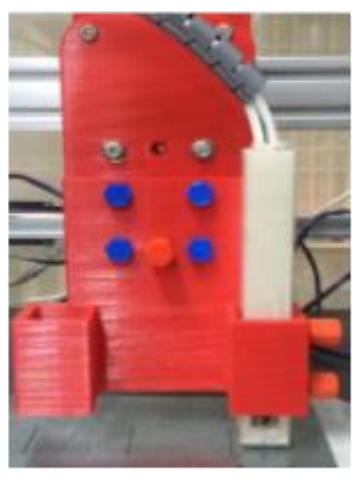

Figure 3. (a) Position illustration of two AMR sensors, (b) The picture of the developed ECT from bottom view and (c) Position of eddy current sensor probe on carbon steel plate

Table 1. Specifications of the ECT probe

\begin{tabular}{cc}
\hline Specifications & Setting \\
\hline Exciting coils & 60 turns square coil \\
& $0.1 \mathrm{~mm}$ diameter of coil \\
Copper wire $(\mathrm{Cu})$ \\
Size of probe & $11.5 \mathrm{~mm} \times 11.5 \mathrm{~mm}$ \\
XY stage & Stepper motor resolution: $0.1 \mathrm{~mm}$ \\
Current & Maximum size: $45 \mathrm{~cm} \times 60 \mathrm{~cm}$ \\
Lift Off & $4 \mathrm{~mA}$ \\
& $1 \mathrm{~mm}$ \\
\hline
\end{tabular}

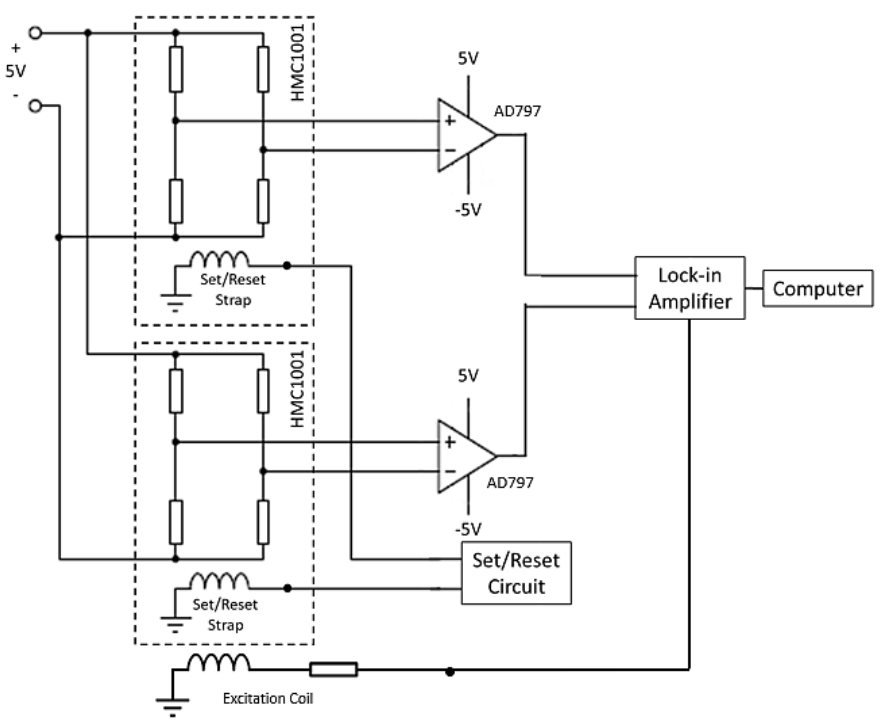

Figure 4. Schematic diagram of the developed ECT probe

\section{Measurement System}

The ECT probe and its experiment setup are shown in Figure 5. The diameter of the coil is $0.1 \mathrm{~mm} \mathrm{Cu}$ wire with 60 turn square coil is used to generate the excitation magnetic field. The probe scanned on top of the sample using XY-stage with a maximum range of scanning area which is $45 \mathrm{~cm} \times 60 \mathrm{~cm}$. 
The AMR output signal will be detected through the lock-in amplifier and the magnetic field distribution of the sample will be captured and recorded in LabVIEW. The lock-in amplifier will give two outputs which are the magnitude of real (in-phase) and imaginary (out of phase) components of the detected magnetic responses. The image mapping of the magnetic response characteristics will be constructed in LabVIEW and the result will be analyzed to identify the property of the flaw.

For the scanning mechanism, an $X Y$ stage is used and a sample with a maximum dimension of $45 \mathrm{~cm} \times 60 \mathrm{~cm}$ can be scanned. By using the $X Y$ stage, the position of ECT probe can be determined with 0.1-mm resolution of stepper motor. The material of the stage is made from aluminium frames and operates at a power voltage of DC $12 \mathrm{~V}$. The $X Y$ stage consists of 3 stepper motors which is one motor for the $X$-axis and the rest are for the $Y$-axis. Each motor is driven by an Arduino Nano microcontroller and operates as intended. The output signal that is captured by the probe must be synchronized to get a reliable result. Before the experiment starts, the probe must be placed at the right position to obtain the data of the sample. The probe will pick up the data at each point according to the motion of the $X Y$ stage. When all the data are successfully taken, the collected data will produce a coordinate-based list of magnetic responses.

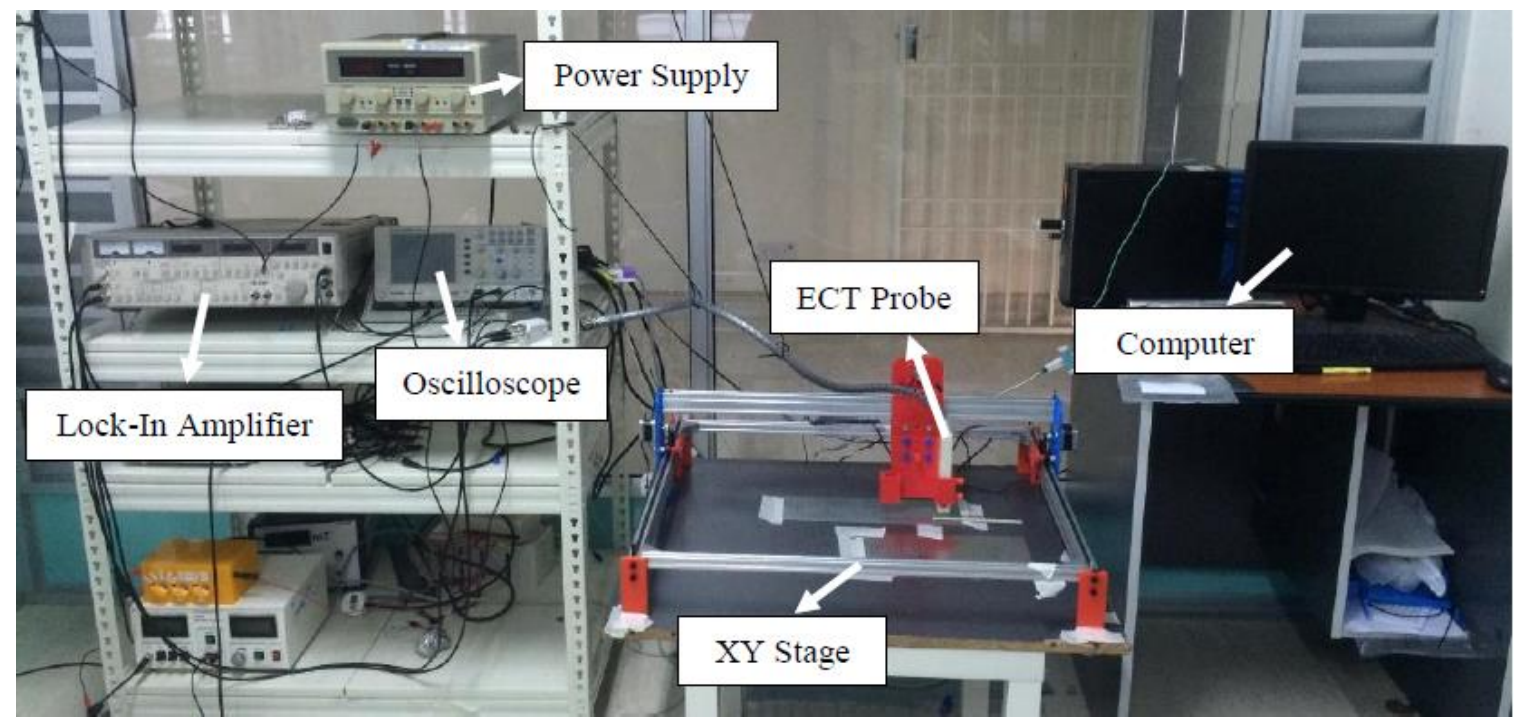

Figure 5. The picture of ECT probe and its experiment setup

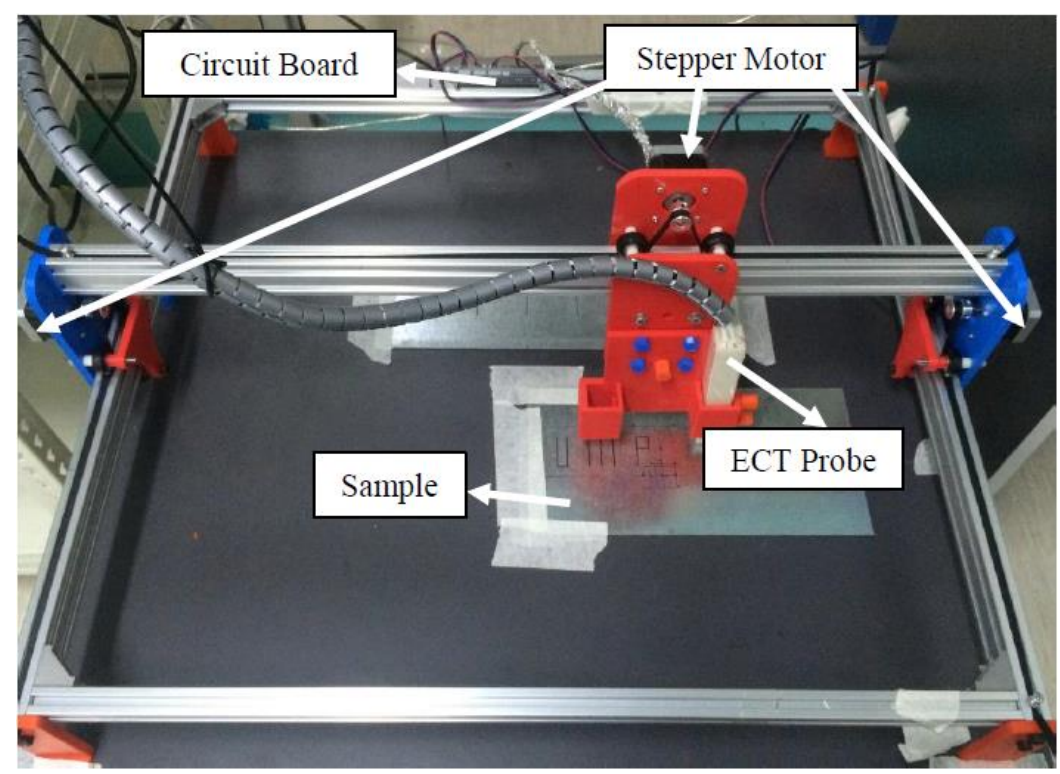

Figure 6. Image of $X Y$-stage

\section{RESULTS AND DISCUSSION}

\section{Line Scanning of Slits with Different Depth}

\section{Comparison of Different Detection Technique}

In this section, different detection techniques were studied to investigate the characteristic of the output signal of each technique. The magnetic response from each slit was measured by doing a line scanning measurement where the probe 
moved horizontally across the slits on the prepared carbon steel plate. The excitation field was set to be $1 \mathrm{kHz}$ and generated by a 4-mA current. Figure 7 shows the output signal of different detection techniques. Figure 7 (a) shows the absolute measurement of the magnetic field response $B z$ from only one AMR sensor using a constant phase of the reference signal. Although the signals showed clear intensity changes at the crack area, the detected signals showed a drift characteristic of the offset signal.

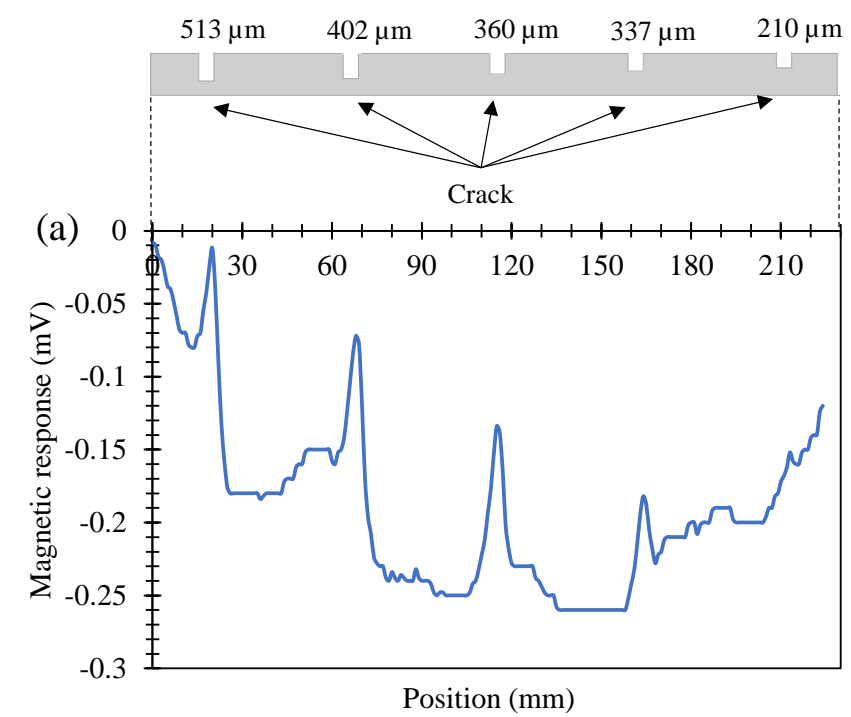

(b)
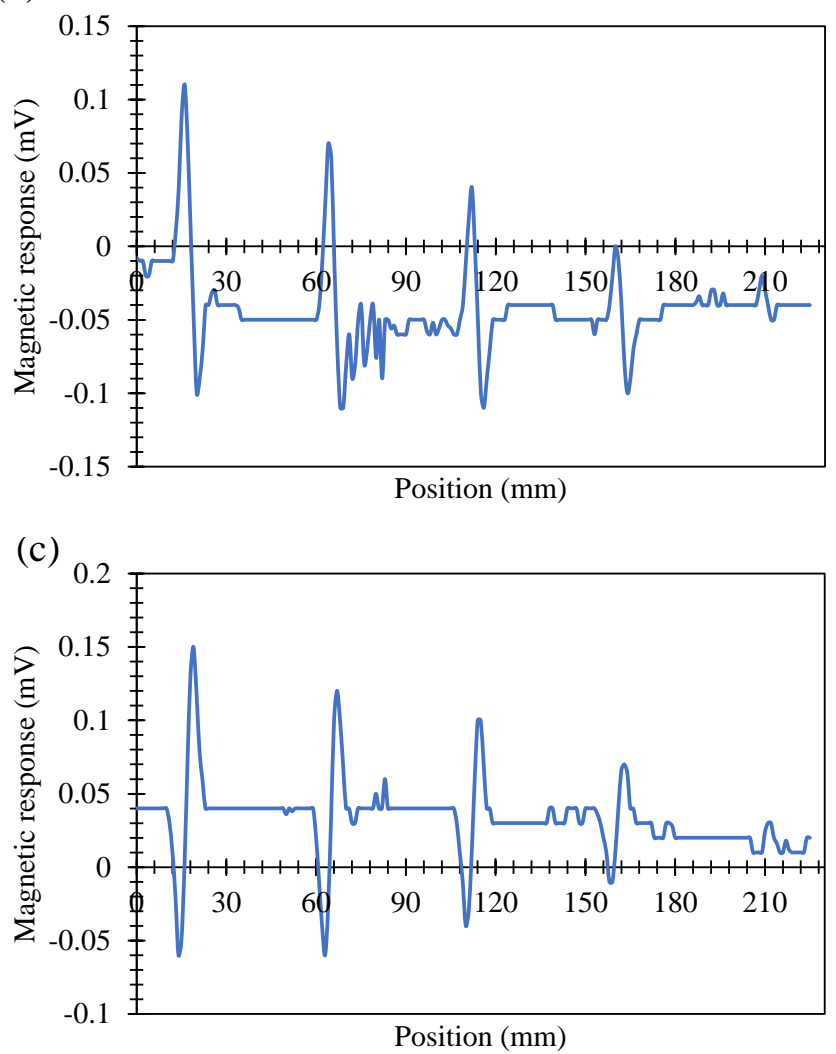

Figure 7. Line-scanned magnetic field intensity with differential technique, (a) Absolute measurement $B_{z}$ of the magnetic response with a constant phase of the reference signal, (b) Differential measurement $d B_{z} / d_{x}$ of the magnetic response with a fix location-based phase referencing technique and (c) Differential measurement $d B_{z} / d_{x}$ of the magnetic response with a sensor-based phase referencing technique

To reduce the drift, a differential technique could be used. Figure 7 (b) shows the result of the intensity of the differential vector (sensor 1 - sensor 2) $d B_{z} / d_{x}$ using a fix location-based phase of the reference signal when the probe was moved across the slits. The signal still showed lots of noise and the signal intensity decreased. It was hard to localize the slits if there was too much of noise in the detected signals. It was important to have a good reference so that the slits 
area could be detected clearly and easily. To reduce this effect, a new phase referencing technique was proposed where the phase of one AMR sensor was used as the reference signal.

This sensor would act as a dynamic phase referencing technique where the phase would deviate depending on the location, thus minimizing the effect of inhomogeneous magnetic properties across a finite area of the sample. The performance of this technique is shown in Figure 7 (c). The drift characteristic was significantly reduced and able to detect the slit-induced signal. Therefore, the differential technique was the best technique compare to the others with the condition of the required reference signal must be dynamic from one point to one point.

\section{Magnetic Response Distribution with Different Depth}

After the line scan measurement, the probe was used to map the magnetic distribution around the slit. In this section, a comparison of magnetic distribution has been made with the different detection techniques. The aim was to gain the best output signal based on the localization performance of slits. Similar with the line scanning measurement, excitation field for 2-D mapping also was set to be $1 \mathrm{kHz}$ and generated by a 4-mA current. The probe has measured the sample in the region of $25 \mathrm{~mm} \times 50 \mathrm{~mm}$ where the slit was located at the red dotted line as shown in Figure 8 .

(a)

(i)

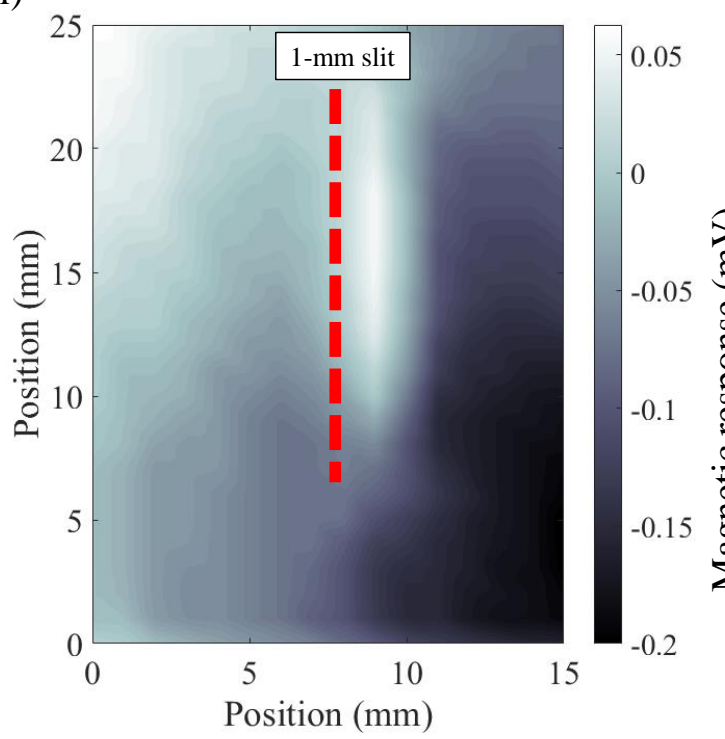

(ii)

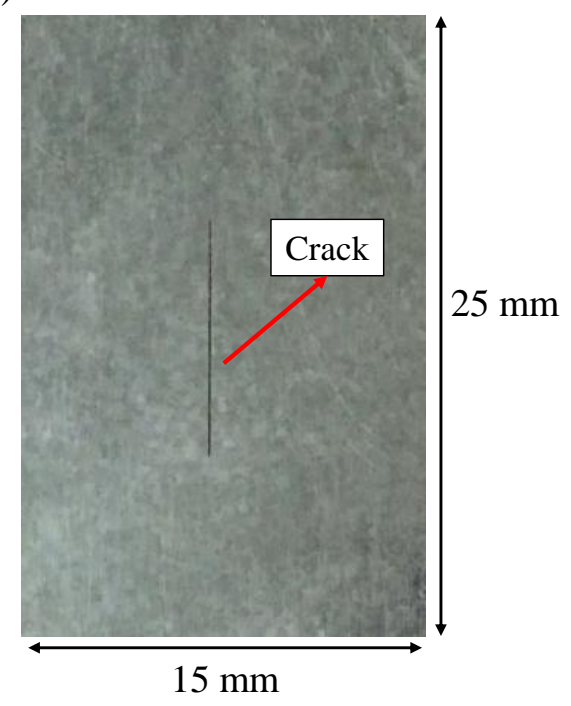

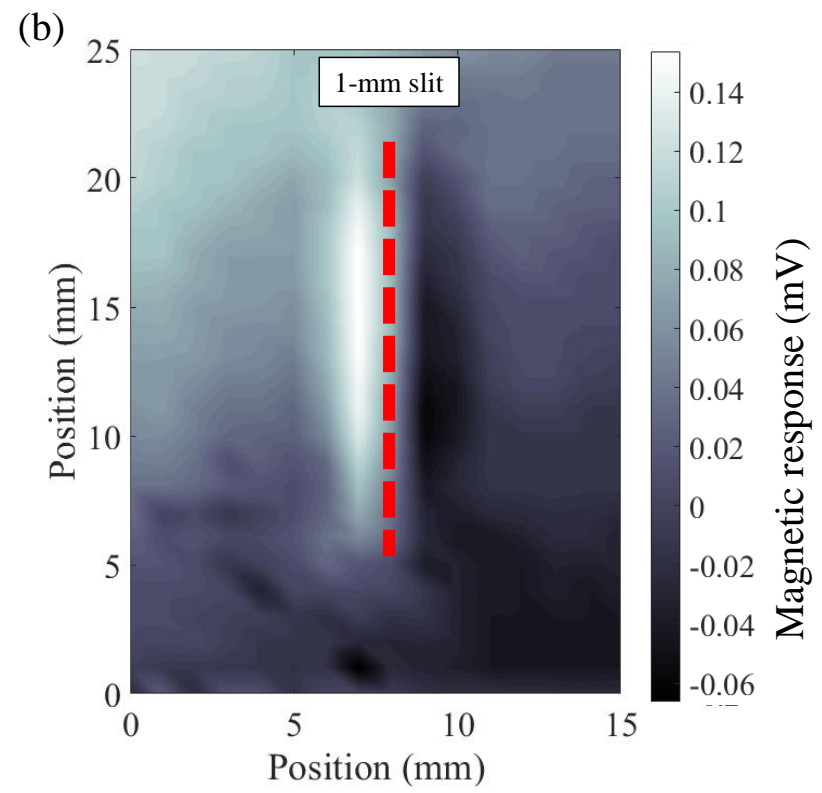




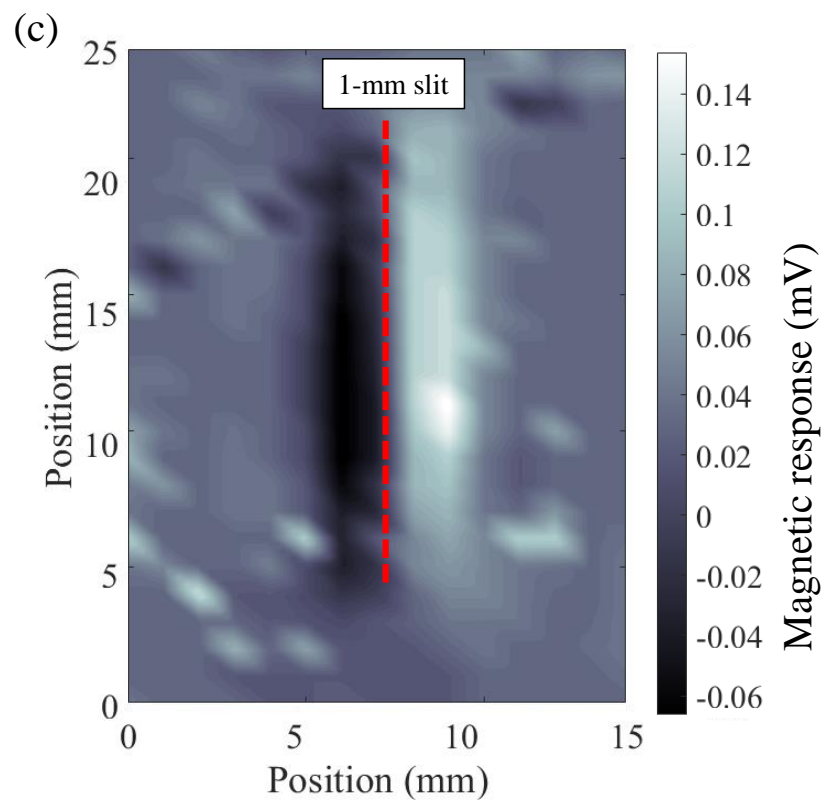

Figure 8. 2-D magnetic map of the slit with 513- $\mu$ m depth: (a) (i)Absolute measurement $B_{z}$ of the magnetic response with a constant phase of the reference signal, (ii) Artificial slit of depth $513 \mu \mathrm{m}$ on a galvanized carbon steel plate, (b) Differential measurement $d B_{z} / d_{x}$ of the magnetic response with a fix location-based phase referencing technique and (c)

Differential measurement $d B_{z} / d_{x}$ of the magnetic response with a sensor-based phase referencing technique

Figure 8 (a) shows changes of intensity around the slit area but it was difficult to expect where was the precise location of the slit since this technique did not applied differential technique. The signal showed lots of noise and it is hard to recognize the position of the slit since there was too much noise signal detected. Figure 8 (b) shows the result of the magnetic distribution using a differential technique with a fixed location-based as the reference. The result is substantially better compare to Figure 8 (a), the intensity changes were still visible in the presence of the slit, but the noise can still be detected. This due to the reference signal did not change, therefore the output signal was not clear, and difficult to identify the slit area. Similar to the line scanning measurement, a good reference is needed to apply a new phase referencing technique so that the slit could be detected effortlessly. Therefore, by implanting the differential technique using the sensor-based phase as the reference signal, the noise signal can be diminished. The effectiveness of this technique is shown in Figure 8 (c) where the intensity changes due to the presence of slits could be seen. The analysis leads to the following conclusion where the differential measurement of the magnetic response with a sensor-based phase referencing technique was the finest approach to identify a crack in steel plate.

\section{CONCLUSION}

This paper proposed an ECT probe for non-destructive testing of metal materials using different detection techniques. The probe consists of an excitation coil to produce a magnetic field and high sensitivity AMR sensors. The ECT probe was fabricated and tested by inspecting the defects in the carbon steel plate. Three different techniques were compared and evaluated by the magnetic response measurement. From all the techniques, it is obvious that the differential technique of the magnetic response with a sensor-based phase referencing has better detectability of the presence of the defect at the excitation frequency $1 \mathrm{kHz}$. In order to represent the comparison in the magnetic response waveforms, a percentage average value was used to represent the comparison. By simply comparing these three techniques, the differential technique of the magnetic response with a sensor-based phase referencing indicate $83 \%$ whereas $78 \%$ for magnetic response with a fix location-based phase referencing technique and 39\% for the magnetic response with a constant phase of the reference signal. The magnetic distribution results also illustrated that the differential technique revealed clear signal intensity change around the slit area. This allows the conclusion that the developed probe is a promising tool to detect flaws in carbon steel plates using differential techniques. Future investigations for different types of cracks are necessary to validate the kinds of conclusions that can be drawn from this study.

\section{ACKNOWLEDGMENT}

Funding for the present work was provided by the Research Management Center of Universiti Malaysia Pahang under grant number of RDU1903100 and Ministry of Higher Education of Malaysia under grant number of FRGS/1/2019/TK04/UMP/02/4 (RDU1901154). 


\section{REFERENCES}

[1] J. Hola and K. Schabowicz, "State-of-the-art non-destructive methods for diagnostic testing of building structures - anticipated development trends," Arch. Civ. Mech. Eng., vol. 10, no. 3, pp. 5-18, 2010. doi: 10.1016/S1644-9665(12)60133-2

[2] J. García-Martín, J. Gómez-Gil, and E. Vázquez-Sánchez, "Non-destructive techniques based on eddy current testing,” Sensors, vol. 11, no. 3, pp. 2525-2565, 2011. doi: 10.3390/s110302525

[3] G. Sposito, C. Ward, P. Cawley, P. B. Nagy, and C. Scruby, "A review of non-destructive techniques for the detection of creep damage in power plant steels," NDT E Int., vol. 43, no. 7, pp. 555-567, 2010. doi: 10.1016/j.ndteint.2010.05.012

[4] T. Sasayama, T. Ishida, M. Matsuo, and K. Enpuku, "Thickness measurement of an iron plate using low-frequency eddy current testing with an HTS coil," IEEE Trans. Appl. Supercond., vol. 26, no. 5, pp. 1-5, 2016. doi: 10.1109/TASC.2016.2535366

[5] D. Mercier, J. Lesage, X. Decoopman, and D. Chicot, "Eddy currents and hardness testing for evaluation of steel decarburizing," NDT E Int., vol. 39, no. 8, pp. 652-660, 2006. doi: 10.1016/j.ndteint.2006.04.005

[6] K. Tsukada, M. Yoshioka, Y. Kawasaki, and T. Kiwa, "Detection of back-side pit on a ferrous plate by magnetic flux leakage method with analyzing magnetic field vector," NDT E Int., vol. 43, no. 4, pp. 323-328, 2010. doi: 10.1016/j.ndteint.2010.01.004

[7] H. Hashizume et al., "Numerical and experimental analysis of eddy current testing for a tube with cracks," IEEE Trans. Magn., vol. 28, no. 2, pp. 1469-1472, 1992. doi: 10.1109/20.123973

[8] S. Tumanski, "Induction coil sensors-A review," Meas. Sci. Technol., vol. 18, no. 3, pp. R31-R46, 2007. doi: 10.1088/09570233/18/3/R01

[9] D.-G. Park, C. S. Angani, B. P. C. Rao, G. Vértesy, D.-H. Lee, and K.-H. Kim, "Detection of the subsurface cracks in a stainless steel plate using pulsed eddy current," J. Nondestruct. Eval., vol. 32, no. 4, pp. 350-353, 2013. doi: 10.1007/s10921-013-01886

[10] K. Tsukada, M. Hayashi, Y. Nakamura, K. Sakai, and T. Kiwa, "Small eddy current testing sensor probe using a tunneling magnetoresistance sensor to detect cracks in steel structures," IEEE Trans. Magn., vol. 54, no. 11, pp. 1-5, 2018. doi: 10.1109/TMAG.2018.2845864

[11] T. Chady, M. Enokizono, and R. Sikora, "Crack detection and recognition using an eddy current differential probe," IEEE Trans. Magn., vol. 35, no. 3, pp. 1849-1852, 1999. doi: 10.1109/20.767393

[12] B. A. Auld, J. C. Moulder, S. Jefferies, P. J. Shull, S. Ayter, and J. Kenney, "Eddy-current reflection probes: Theory and experiment," Res. Nondestruct. Eval., vol. 1, no. 1, pp. 1-11, 1989. doi: 10.1007/BF01607866

[13] N. A. Nadzri, M. Ishak, M. M. Saari, and A. M. Halil, "Development of eddy current testing system for welding inspection," in 2018 9th IEEE Control and System Graduate Research Colloquium (ICSGRC), 2018, no. 1, pp. 94-98. doi: 10.1109/ICSGRC.2018.8657511

[14] K. Allweins, M. von Kreutzbruck, and G. Gierelt, "Defect detection in aluminum laser welds using an anisotropic magnetoresistive sensor array,” J. Appl. Phys., vol. 97, no. 10, p. 10Q102, 2005. doi: 10.1063/1.1852391

[15] M. A. Hadi Putera Zaini, M. Mawardi Saari, N. A. Nadzri, A. Mohd Halil, and K. Tsukada, “An MFL Probe using shiftable magnetization angle for front and back side crack evaluation," Proc. - 2019 IEEE 15th Int. Colloq. Signal Process. its Appl. CSPA 2019, pp. 157-161, 2019. doi: 10.1109/CSPA.2019.8696064

[16] K. Tsukada, T. Kiwa, T. Kawata, and Y. Ishihara, "Low-frequency eddy current imaging using MR sensor detecting tangential magnetic field components for nondestructive evaluation," IEEE Trans. Magn., vol. 42, no. 10, pp. 3315-3317, 2006. doi: 10.1109/TMAG.2006.879754

[17] K. Tsukada et al., "Integrated magnetic sensor probe and excitation wire for nondestructive detection of submillimeter defects," IEEE Magn. Lett., vol. 10, pp. 1-5, 2019. doi: 10.1109/LMAG.2019.2936392 